\title{
Cayenne aspiration: an unusual type of lower airway foreign-body aspiration
}

This article was published in the following Dove Press journal:

Clinical Interventions in Aging

21 November 2014

Number of times this article has been viewed

\author{
Lianjun Lin' \\ Yuchuan Wang' \\ Xiankui Zha ${ }^{2}$ \\ Fei Tang ${ }^{2}$ \\ Liping Lv ${ }^{2}$ \\ Xinmin Liu' \\ 'Geriatric Department, Peking \\ University First Hospital, Beijing, \\ 2Pulmonary Intervention Department, \\ Anhui Chest Hospital, Hefei, People's \\ Republic of China
}

Purpose: Cayenne aspiration is an unusual type of foreign-body aspiration that is usually
misdiagnosed. This article analyzes the clinical features of cayenne aspiration in the lower
airway. Patients and methods: Clinical data on eight adult patients with cayenne aspiration were retrospectively analyzed. Six were elderly patients. The data were collected from Peking University First Hospital and Anhui Chest Hospital between January 2010 and August 2014.

Results: The most common symptoms of cayenne aspiration were cough (eight cases, 100\%) and sputum (five cases, $62.5 \%$ ). Only one patient (12.5\%) could supply the history of aspiration on his first visit to doctor and was diagnosed definitely without delay. The other seven cases were misdiagnosed as pneumonia and the time to accurate diagnosis was from 1 month to 6 months. The history of aspiration could be recalled after confirmed diagnosis for the other seven cases. The most common presentation shown by chest computed tomography (CT) was pneumonic opacity (eight cases, 100\%). The existence of cayenne could not be detected by chest CT in any of the patients. All the patients were diagnosed definitively and managed successfully with flexible bronchoscopy. Cayenne was more often lodged in the right bronchus tree (seven cases, $87.5 \%$ ), especially the right lower bronchus (four cases, $50 \%$ ). The segment of cayenne was complete in five cases (62.5\%) and scattered in three cases $(37.5 \%)$.

Conclusion: The clinical features of cayenne aspiration are usually obscure and nonspecific which may lead to delay in diagnosis. Flexible bronchoscopy is safe and useful for early diagnosis and effective management.

Keywords: clinical features, flexible bronchoscopy, chest CT, pneumonia

\section{Introduction}

Foreign-body aspiration into the airway is harmful and even life threatening. ${ }^{1}$ Foreignbody aspiration occurs more often in children but its incidence in, and damage to, adults cannot be ignored. In adults, foreign-body aspiration often occurs in elderly people and its incidence increases with aging. ${ }^{1,2}$ The most common type of material aspirated into the lower airway is organic substance, in particular, bone fragments. ${ }^{1,3,4}$ The nature of the aspirated foreign body has been proposed to be different in Asian and Western adults. ${ }^{2,5}$

Chinese people have a fondness for eating hot and spicy food with tasty cayenne pepper. Cayenne aspiration into the lower airway is an unusual type of foreign-body aspiration in adults and, to the best of our knowledge, has never been reported. In the past few years, we diagnosed eight cases of cayenne aspiration, most of which were in elderly patients and there was a different period of delay to accurate diagnosis in each. For these patients, the history of cayenne aspiration was not obvious, the symptoms were not specific, the presentation of chest computed tomography (CT) was obscure, and the application of flexible bronchoscopy was delayed. 
The peppery and hot diet has become more popular in the People's Republic of China and there is an increased risk of aspiration of cayenne pepper. The early recognition and suitable treatment of cayenne aspiration is of vital importance. Herein, we report the cases of cayenne aspiration for better understanding of the clinical features of the rare condition.

\section{Materials and methods}

\section{Study design}

We analyzed the medical data of patients with cayenne aspiration in Peking University First Hospital and Anhui Chest Hospital between January 2010 and August 2014. Patients with definitely diagnosed cayenne aspiration into the lower airway were recruited. The symptoms, aspiration history, chest CT image presentation, and treatments were reviewed. The features and lodgment site of the cayenne were recorded.

\section{Endoscopic procedures}

Flexible bronchoscopes were used in all patients. Topical anesthesia was given intranasally using lidocaine. The equipment used to remove the foreign bodies were foreign-body forceps and biopsy forceps.

\section{Data analysis}

The data of the patients, including age, sex, symptoms, chest CT image, process of diagnosis and treatment, and endoscopic findings including the features and locations of cayenne, were collected and analyzed.

\section{Results}

\section{Clinical manifestations}

Four of the eight patients were male. The range of age was from 41 to 75 years with a median age of 68 years. Six of the patients were older than 65 years. The most common symptoms were cough (eight cases, 100\%) and sputum (five cases, 62.5\%). One patient (12.5\%) reported fever. Only one patient $(12.5 \%)$ could supply the history of cayenne aspiration on his first visit to the doctor and was diagnosed definitively without delay. The other seven patients could not supply the history of aspiration on their initial visits to doctors. They were misdiagnosed with pneumonia and accepted antibiotic therapy. The time range of delay to accurate diagnosis was from 1 month to 6 months. The average time of delay to accurate diagnosis was 2.8 months. Interestingly, all seven patients could recall the history of cayenne aspiration after confirmed diagnosis by flexible bronchoscopy.

\section{Chest computed tomography}

The most common presentation of cayenne aspiration on chest CT was pneumonic consolidation or opacity (eight cases, 100\%). Cayenne pepper could not be identified by chest $\mathrm{CT}$ in any of the patients. Other findings on chest CT included atelectasis (two cases, 25.0\%), obstructive pneumonia (one case, 12.5\%), and bronchial stenosis (one case, $12.5 \%)$.

\section{Foreign body}

The cayenne was most often lodged in the right bronchus tree (seven cases, $87.5 \%$ ), especially the right lower bronchus (four cases, 50\%). The location of the cayenne is shown in Table 1. The segment of cayenne was complete in five cases (62.5\%) and scattered in three cases (37.5\%). As shown in Figure 1F, the aspirated cayenne may be in tiny fragments after chewing by teeth or in one big segment with tiny fragments (Figure 3C). It can also be dispersed as two relatively big segments lodged in two bronchial lumens (Figure 3F).

\section{Flexible bronchoscopy}

Flexible bronchoscopy was performed in all the patients and revealed the cayenne lodged in the bronchus (Figure 2). The mucosa surrounding the cayenne was severely inflamed and hypertrophic with purulent secretion. In three cases (37.5\%), follicular change of the mucosa, granulation tissue hyperplasia, and different degrees of luminal stenosis were observed (Figure 2, Cases 1, 3, and 6). Flexible bronchoscopy removed the cayenne successfully in all the patients.

It is worth pointing out that the cayenne was distributed as tiny fragments in one case (12.5\%) (Figure 1E) and it was important to remove all the fragments completely and carefully. In another case, the patient aspirated two big segments of cayenne, which lodged in two different bronchial lumens (Figure 2, Case 4 and Figure 3F). Careful and thorough examination under bronchoscopy was essential and crucial to make sure that all parts of the cayenne had been removed.

Table I The location and frequency of cayenne in the bronchial tree

\begin{tabular}{ll}
\hline Site & Case(s), $\mathbf{n}(\%)$ \\
\hline Right & \\
Bronchus intermedius & $2(25.0)$ \\
Middle lobe & $\mathrm{I}(12.5)$ \\
Lower lobe & $4(50.0)$ \\
Left & \\
Lower lobe & $\mathrm{I}(12.5)$ \\
\hline
\end{tabular}




\section{Case 1}

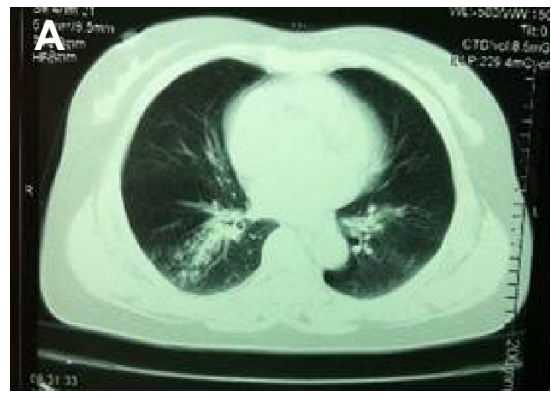

Case 2

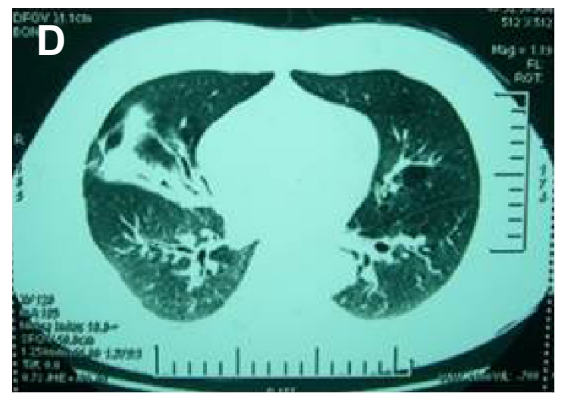

\section{Case 3}

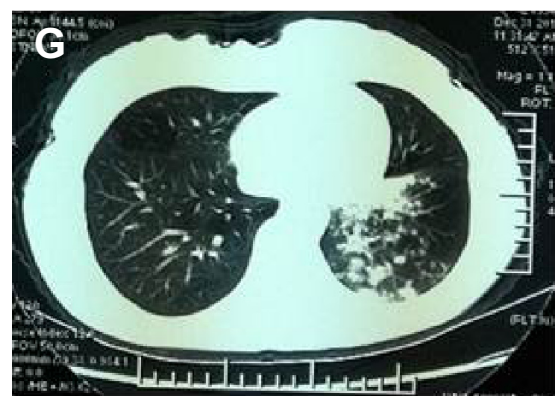

\section{Case 4}

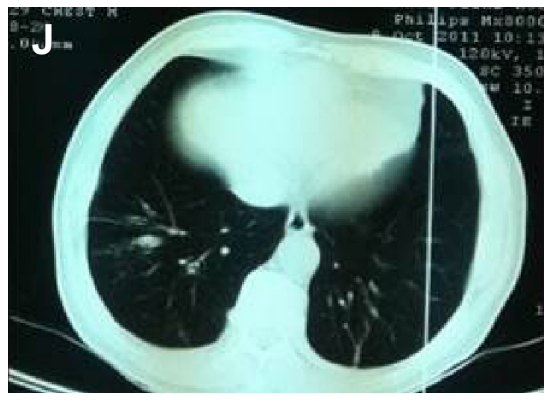

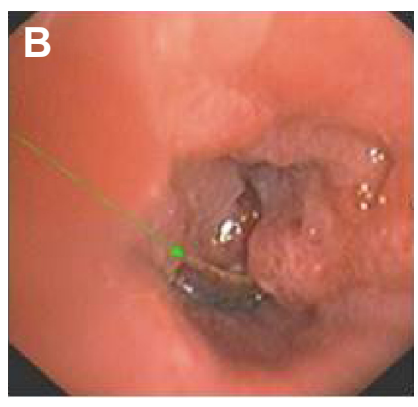
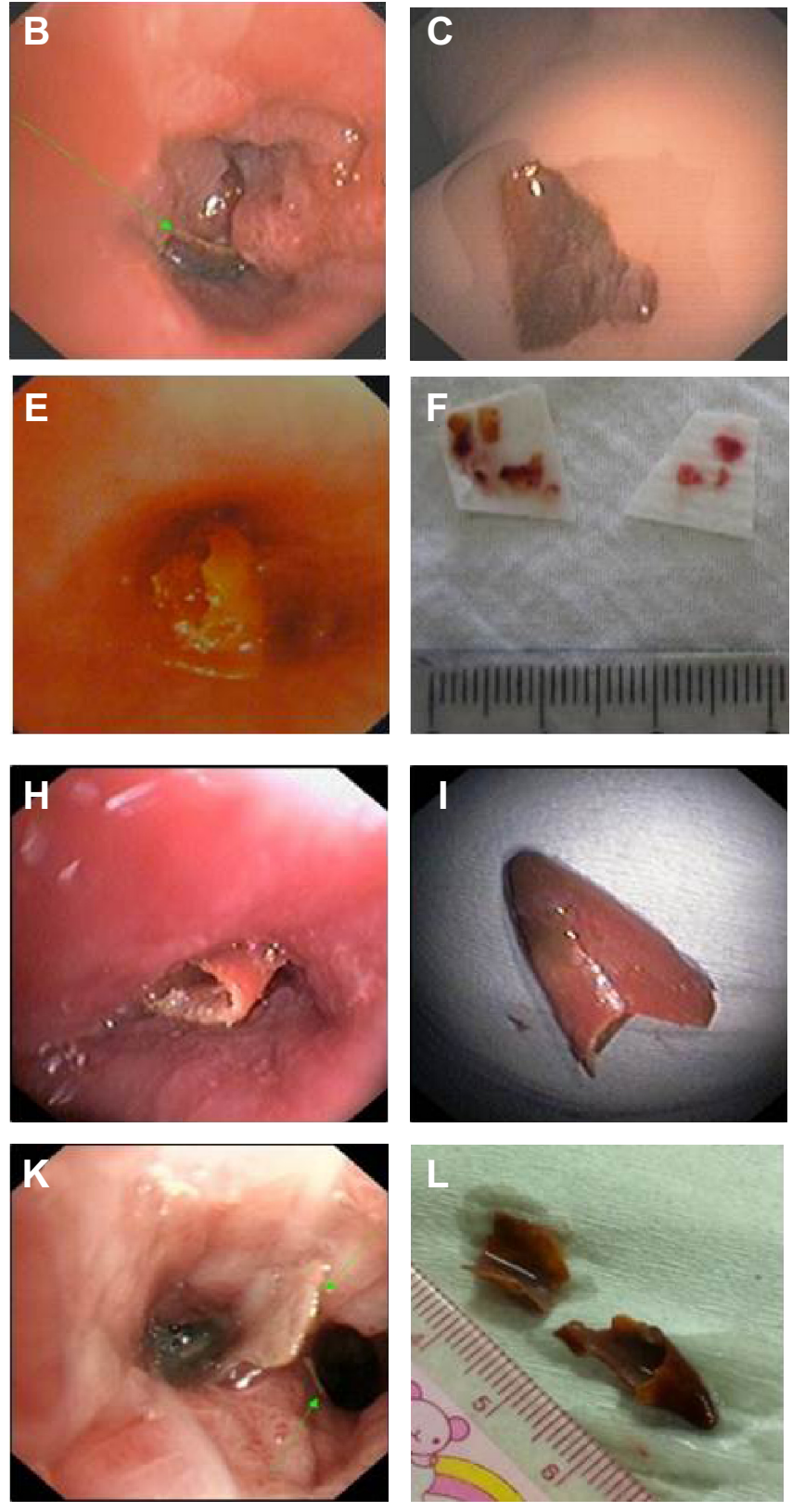

Figure I Presentation under chest computed tomography (A, D, G, and J) and bronchoscopy (B, E, $\mathbf{H}$, and $\mathbf{K})$, and the cayenne removed from patients (C, F, I, and $\mathbf{L})$. Note: The green arrow in $\mathbf{B}$ and $\mathbf{K}$ indicates the less obvious segments of cayenne.

No management-related complications were observed. The pathology of the removed foreign body showed evidence of plant cells (Figure 3D and E). The biopsy of the bronchial mucosa further excluded the possibility of tumor.

\section{Discussion}

Foreign-body aspiration into the lower airway is rare in adults and accounts for about $20 \%$ of the reported cases. However, its incidence increases with advancing age and increases steadily with age from the sixth decade on. ${ }^{6}$ It can be a serious medical problem and is associated with morbidity and mortality. The symptoms can vary from life-threatening airway obstruction to nonspecific symptoms. The majority of foreign bodies aspirated are food, primarily bone. ${ }^{6}$ The nature of the aspirated foreign bodies varies across different countries and regions due to different eating habits. Chinese people have different eating patterns to Western people, resulting in different aspirated foreign bodies. ${ }^{7,8}$

In the People's Republic of China, the peppery and hot diet has become more and more popular and fashionable in recent years. Chinese people have a fondness of eating peppery food with cayenne. Cayenne may be used variously 


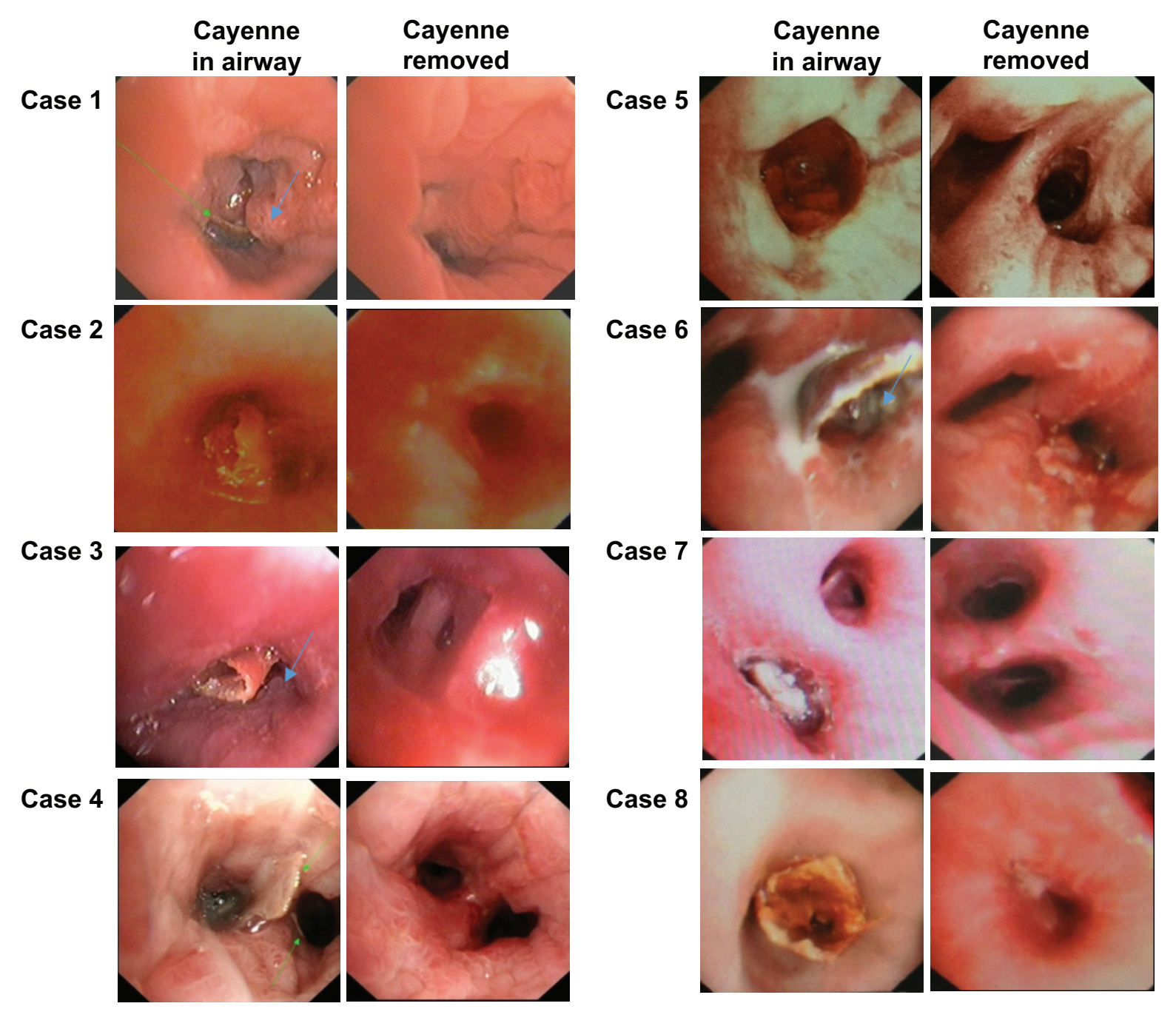

Figure 2 Presentation under bronchoscopy before and after removal of the aspirated cayenne.

Notes: The green arrow in Case I and Case 4 indicates the less obvious segments of cayenne. The blue arrow in Cases I, 3 , and 6 indicates the follicular change of the mucosa, granulation tissue hyperplasia, and different degrees of luminal stenosis.

in its fresh form, dried and powdered, or as dried flakes as ingredients to improve the taste of food. Cayenne is not only a delicious ingredient but also a potentially dangerous foreign body that may be aspirated into the airway. In the past few years, we diagnosed eight cases of cayenne aspiration and most of them were in elderly patients. In most of these patients, diagnosis was delayed for months, which led to various degrees of complication.

In our study, we found that the clinical features of cayenne aspiration into the airway are usually obscure, which may cause delay in diagnosis. In particular, the non-specificity of symptoms, the absence or the ignorance of aspiration history, the lack of direct evidence of the existence of cayenne on chest $\mathrm{CT}$, and the delayed application of bronchoscopy were the probable reasons for misdiagnosis. These are discussed following.
Clinical manifestations of foreign-body aspiration into the lower airway are often variable and nonspecific in adults. The site of foreign-body impaction determines the clinical presentation. If the foreign body is lodged into the trachea, inspiratory stridor and bouts of coughing can occur. If the foreign body is aspirated into the bronchi, the symptoms may be variable, and can include cough, sputum, and wheezing. Untreated or unrecognized cayenne aspiration can result in obstructive pneumonitis, atelectasis, or airway hyperresponsiveness. Our study showed that a sudden onset of coughing was common in all the cases and may last for several months, accompanied by sputum or not. This was probably due to the active ingredients of cayenne, which can induce chronic respiratory symptoms, including cough and dyspnea. For example, capsaicin, a neurotoxin derived from chili peppers, can cause shortness of breath and cough.,10 

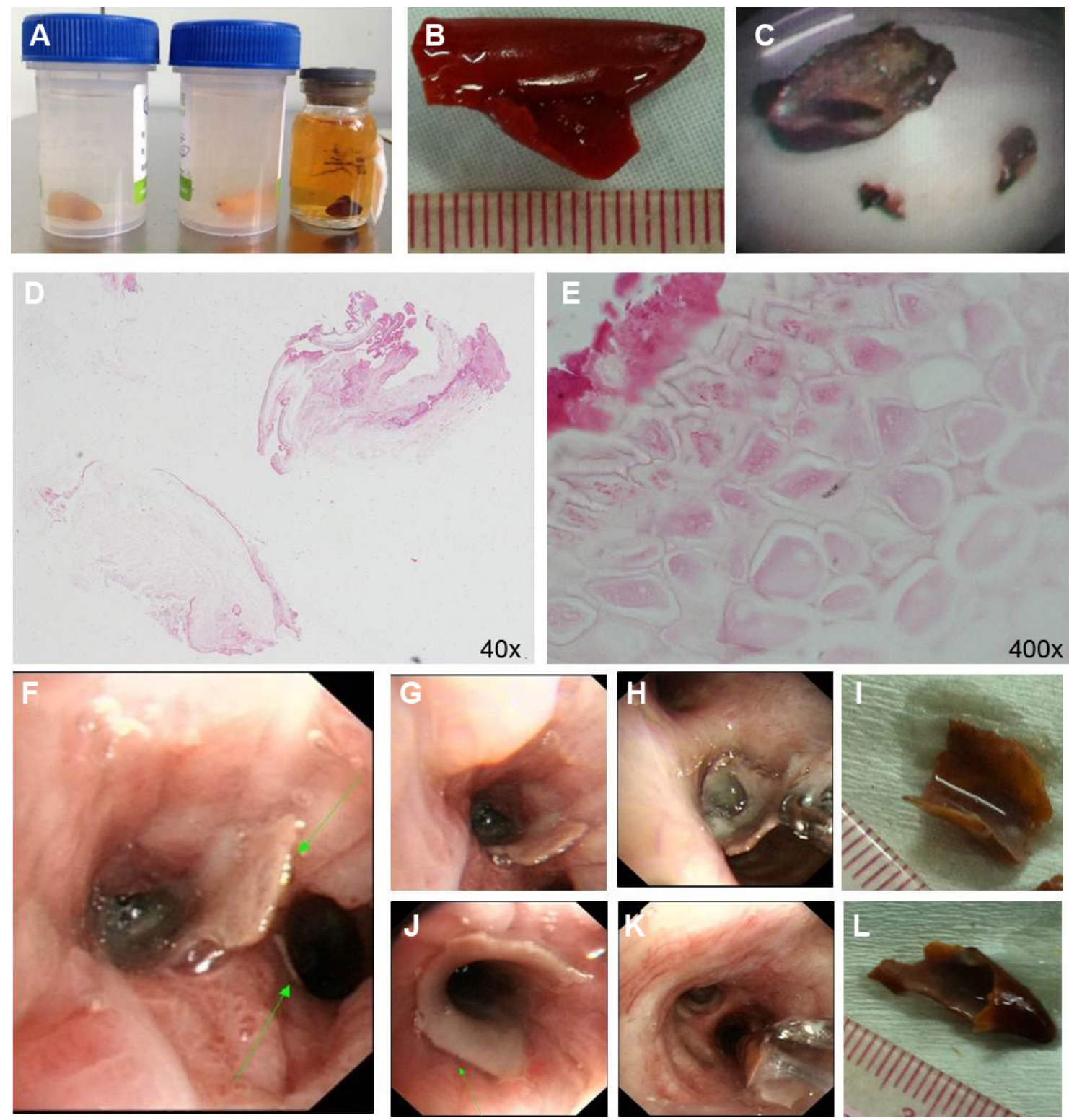

Figure 3 (A-C) The appearance of the cayenne in patients, (D, E) pathology of the foreign body, and (F) the presentation under bronchoscopy of Case 4 . The different parts of cayenne were lodged in different bronchial lumens $(\mathbf{G}, \mathbf{J})$ and were removed by bronchoscopy $(\mathbf{H}, \mathbf{K})$. (I, L) The different parts of cayenne removed by bronchoscopy. Notes: The green arrows in (F) and $(\mathbf{J})$ indicate the segments of cayenne.

Animal experiments indicate that the ingestion of less than $40 \mathrm{~g}$ of capsaicin may be fatal. ${ }^{11}$

Because the cayenne was soft and hollow, the taper-like cayenne or tiny fragments were seldom lodged in the tracheal or main bronchus, so the classic triad of cough, wheezing, and choking in foreign-body aspiration was not observed in our study. The symptoms of cough and sputum associated with cayenne aspiration may be obscure, nonspecific, and mimic those of other respiratory diseases such as pneumonia, bronchitis, or asthma. Thus, nonspecific respiratory symptoms may be mistakenly attributed to other medical conditions, unless there is a clear history of aspiration of cayenne.
Nonspecific symptoms may contribute little toward confirmation of diagnosis of airway aspiration, so aspiration history should be investigated carefully. In our study, only one patient could confirm the history of aspiration on his first visit to the doctor and received a definite diagnosis and immediate management under bronchoscopy. What was interesting and thought provoking was that all the other seven patients who did not initially supply history of cayenne aspiration could recall the history of aspiration clearly and accurately after the confirmed diagnosis by bronchoscopy. The low proportion of recognition of cayenne aspiration was probably due to the ignorance or neglect of aspiration event. 
The aspiration event might be covered up by an accompanying cough that was mistakenly attributed to the stimulating effect of cayenne. Because cayenne was soft and thin, the aspiration event may be difficult to be noticed. If their doctors had been alert to foreign-body aspiration during their history taking, the aspiration history could probably have been collected. Careful inquiry is helpful for early diagnosis. Both doctors and patients should pay more attention to the history of aspiration of foreign body.

Generally speaking, if the aspiration history is not suggestive, chest CT may assist in detecting the foreign body. ${ }^{12} \mathrm{As}$ shown in another study by our group, chest CT can demonstrate the existence of a foreign body directly in about $30 \%$ of patients. ${ }^{4}$ The common presentations of foreign-body aspiration include pneumonic patch, atelectasis, high-density lesion in airway, pleural effusion, space-occupying lesion, and lung abscess. ${ }^{4}$ In the present study, the CT findings in patients with cayenne aspiration were indirect and nonspecific. Pneumonic consolidation or opacity was the most common finding. This was probably due to the physical features of cayenne. The positive detection rate by $\mathrm{CT}$ is largely dependent on the physical properties of the aspirated material and the slice thickness relative to the size of the foreign body. Metal or bone, which is radiopaque, is easier to detect on chest CT. Plant material is less likely to be detected directly by chest CT. A chest CT scan that does not provide evidence of the direct existence of a foreign body should never be accepted as proof that there is no foreign body in the airway. Direct visualization of the airways by bronchoscopy is important.

Flexible bronchoscopy is effective both in the diagnosis and removal of aspirated cayenne. In our study, most of the patients had been misdiagnosed with pneumonia for months until the aspiration of cayenne was confirmed through flexible bronchoscopy. First, although cayenne may be added to food variously in its fresh form, dried and powdered, or as dried flakes, in our study, only cayenne in fresh form was observed. This is probably because the dried powder and flakes were not visible under bronchoscopy. Secondly, foreign bodies in the airway tend to localize in the right bronchial tree, as reported in the literature. ${ }^{7}$ Cayenne is no exception. In our study, the cayenne was more often lodged in the right bronchus tree, especially the right lower bronchus. This right-side predominance can be explained by the vertical nature of the right main bronchus, its larger diameter, and the greater airflow through it.

The cayenne aspirated in all the cases was removed by flexible bronchoscopes successfully without complication. Our study shows that the application of flexible bronchoscopy is safe and effective for patients with cayenne aspiration.
Moreover, we should be alert to the distribution and location of cayenne during management with bronchoscopy, because, in some patients, the cayenne was scattered as tiny fragments or different parts lodged in different bronchial lumens. It was important to remove all the fragments completely and carefully. Careful and thorough examination under bronchoscopy was essential and crucial to make sure that all parts of the cayenne were removed.

Gustav Killian performed the first bronchoscopy in 1897 to treat a patient with foreign-body aspiration by esophagoscope. ${ }^{13}$ Since then, bronchoscopy has become a useful and effective practice in the evaluation, diagnosis, and treatment of foreign-body aspiration. With the development and progress of this technique, the mortality rate associated with foreign-body aspiration has decreased dramatically. ${ }^{14,15}$ Due to the high rate of misdiagnosis in patients with cayenne aspiration, flexible bronchoscopy should be performed actively in suspected cases of foreign-body aspiration. ${ }^{16,17}$ Cayenne in the airway can be removed safely and effectively with flexible bronchoscopy by an experienced expert.

\section{Conclusion}

Cayenne aspiration may occur in adult patients, especially the elderly. Cough is the prominent symptom, which is not specific, and aspiration history may be obscure. Chest CT may be helpful but the negative result of the existence of cayenne should not exclude the possibility of cayenne aspiration. Flexible bronchoscopy has been demonstrated to be both safe and effective in the diagnosis and treatment of cayenne aspiration.

\section{Acknowledgment}

This work was supported by National Natural Science Foundation of China (NSFC numbers: 30971313, 81141001, 81270114).

\section{Disclosure}

The authors report no conflicts of interest in this work.

\section{References}

1. Boyd M, Chatterjee A, Chiles C, Chin R Jr. Tracheobronchial foreign body aspiration in adults. South Med J. 2009;102(2):171-174.

2. Limper AH, Prakash UB. Tracheobronchial foreign bodies in adults. Ann Intern Med. 1990;112(8):604-609.

3. Ramos MB, Fernández-Villar A, Rivo JE, et al. Extraction of airway foreign bodies in adults: experience from 1987-2008. Interact Cardiovasc Thorac Surg. 2009;9(3):402-405.

4. Lin L, Lv L, Wang Y, Zha X, Tang F, Liu X. The clinical features of foreign body aspiration into the lower airway in geriatric patients. Clin Interv Aging. 2014;9:1613-1618.

5. Lan RS. Non-asphyxiating tracheobronchial foreign bodies in adults. Eur Respir J. 1994;7(3):510-514. 
6. Hsu WC, Sheen TS, Lin CD, Tan CT, Yeh TH, Lee SY. Clinical experiences of removing foreign bodies in the airway and esophagus with a rigid endoscope: a series of 3217 cases from 1970 to 1996. Otolaryngol Head Neck Surg. 2000;122(3):450-454.

7. Baharloo F, Veyckemans F, Francis C, Biettlot MP, Rodenstein DO. Tracheobronchial foreign bodies: presentation and management in children and adults. Chest. 1999;115(5):1357-1362.

8. Chen CH, Lai CL, Tsai TT, Yee YC, Perng RP. Foreign body aspiration into the lower airway in Chinese adults. Chest. 1997;112(1):129-133.

9. Miller JJ, Skolnick J. Inhalation injury after capsaicin exposure. $J K y$ Med Assoc. 2006;104(3):103-105.

10. Roth VS, Franzblau A. RADS after exposure to a riot-control agent: a case report. J Occup Environ Med. 1996;38(9):863-835.

11. Fuller RW, Dixon CM, Barnes PJ. Bronchoconstrictor response to inhaled capsaicin in humans. J Appl Physiol. 1985;58(4):1080-1084.

12. Zissin R, Shapiro-Feinberg M, Rozenman J, Apter S, Smorjik J, Hertz M. CT findings of the chest in adults with aspirated foreign bodies. Eur Radiol. 2001;11(4):606-611.
13. Zöllner F. Gustav Killian, father of bronchoscopy. Arch Otolaryngol 1965;82(6):656-659.

14. Aytaç A, Yurdakul Y, Ikizler C, Olga R, Saylam A. Inhalation of foreign bodies in children. Report of 500 cases. J Thorac Cardiovasc Surg. 1977;74(1):145-151.

15. de Sousa ST, Ribeiro VS, de Menezes Filho JM, dos Santos AM, Barbieri MA, de Figueiredo Neto JA. Foreign body aspiration in children and adolescents: experience of a Brazilian referral center. J Bras Pneumol. 2009;35(7):653-659. English and Portuguese.

16. Orji FT, Akpeh JO. Tracheobronchial foreign body aspiration in children: how reliable are clinical and radiological signs in the diagnosis? Clin Otolaryngol. 2010;35(6):479-485.

17. Korlacki W, Korecka K, Dzielicki J. Foreign body aspiration in children: diagnostic and therapeutic role of bronchoscopy. Pediatr Surg Int. 2011;27(8):833-837.
Clinical Interventions in Aging

\section{Publish your work in this journal}

Clinical Interventions in Aging is an international, peer-reviewed journal focusing on evidence-based reports on the value or lack thereof of treatments intended to prevent or delay the onset of maladaptive correlates of aging in human beings. This journal is indexed on PubMed Central, MedLine,

\section{Dovepress}

CAS, Scopus and the Elsevier Bibliographic databases. The manuscript management system is completely online and includes a very quick and fair peer-review system, which is all easy to use. Visit http://www.dovepress. com/testimonials.php to read real quotes from published authors. 